Proceedings of the National Conference on Neutron Scattering and the Complementary Methods in the Investigations of the Condensed Phases, Chlewiska 2007

\title{
Diffuse Neutron Scattering in the $\mathrm{Mn}_{0.4} \mathrm{Cu}_{0.6}$ Alloy
}

\author{
J. Jankowska-Kisielińska, K. Mikke \\ AND I. FIJAŁ-KIREJCZYK \\ Institute of Atomic Energy, Świerk, 05-400 Otwock, Poland
}

Dedicated to Professor Jerzy Janik on the occasion of his 80th birthday

The diffuse neutron scattering in the $\mathrm{Mn}_{0.4} \mathrm{Cu}_{0.6}$ alloy was measured in the temperature range $15-390 \mathrm{~K}$ at the 3 -axis neutron spectrometer at MARIA reactor in Świerk. The broad maxima of the neutron intensity were observed in the surrounding of the $(0.5,1,0)$ reciprocal lattice point and other symmetry related points. The effect is similar to that observed in $\mathrm{Mn}-\mathrm{Cu}$ alloys with lower $\mathrm{Mn}$ content at room temperature. Our results are in agreement with the earlier finding that the maxima of the short-range order incommensurate spin density wave scattering are placed at the $(0.5 \pm \delta, 1,0)$ reciprocal lattice point with a linear dependence of $\delta$ on the $\mathrm{Mn}$ concentration. In addition, some commensurate antiferromagnetic short-range order was found.

PACS numbers: 61.05.F-, 75.30.Fv, 75.40.-s, 75.50.Lk

\section{Introduction}

$\mathrm{Mn}_{x} \mathrm{Cu}_{1-x}$ alloys were extensively studied over past 50 years. Spin glass properties were recognised and established first in dilute $\mathrm{Cu}-\mathrm{Mn}$ alloys. Magnetic and atomic short-range order (SRO) was studied in this system over 30 years. The presence of the atomic short-range order (ASRO) and the magnetic order of the type of incommensurate spin density wave (ISDW) was demonstrated for Mn content up to 0.40 [1-15]. More detailed study concerns alloys with the Mn content up to $x=0.25$.

An ASRO increases after aging at 400-500 K. It causes an increase in the freezing temperature $\left(T_{\mathrm{F}}\right)$ and the maximum value of the magnetic susceptibility at $T_{\mathrm{F}}[16,17]$. This effect is most pronounced for Mn concentration range 0.15-0.25.

There is no doubt concerning the presence of the SRO ISDW and its origin. ISDW is formed due to maximum of the magnetic susceptibility for wave-vector 
connecting the flat parts of the Fermi surface [11]. The ISDW wave-vector dimension is connected to the diameter of the Fermi sphere of the $4 s$ electrons. However, there are doubts concerning the role of ISDW for spin-glass state. Some authors $[9,12]$ claim that clusters of the SRO ISDW possess the considerable magnetic moments which are the building blocks of the spin glass. The others [13] claim that magnetic moments forming spin glass do not participate in ISDW, at least for alloys of low Mn concentration. Recently it was corroborated [15] that ferromagnetic chains, oriented along [100]-type directions, are the main peculiarity of the magnetic SRO of the $\mathrm{Cu}_{0.83} \mathrm{Mn}_{0.17}$ alloy. These ferromagnetic chains are formed due to antiferromagnetic interaction between first neighbours and ferromagnetic interaction between second neighbours, connected with the atomic SRO. They can participate both in the ISDW-like magnetic SRO and in the spin glass state.

In more concentrated alloys the spin-glass phenomena are less visible (e.g. smaller and more extended maximum of the susceptibility at $T_{\mathrm{F}}$ ), so the spin-glass properties and the influence of the $\mathrm{SRO}$ on them is easier to study in less concentrated alloys. On the other hand, the evolution of the magnetic SRO and the precursors of the long-range antiferromagnetic order can be studied for the more concentrated alloys. There are only few published data of the diffuse neutron scattering on concentrated, not long-range ordered, $\mathrm{Cu}-\mathrm{Mn}$ alloys [5, 18-20].

Our present investigation on $\mathrm{Mn}_{0.4} \mathrm{Cu}_{0.6}$ alloy were undertaken to give more information about a system where Mn concentration is large but not large enough to create long-range antiferromagnetism. Our main aim was to test the incommensurate type of the SRO antiferromagnetism observed in this alloy [5] and verify the presence of the commensurate magnetic SRO.

Earlier neutron diffuse-scattering data [5] for the alloy with the same composition were claimed to show ASRO and the incommensurate magnetic SRO only. The small angle diffuse scattering was absent so there is no ferromagnetic SRO. In our opinion commensurate antiferromagnetic as well as ferromagnetic SRO can give rise to the diffuse scattering centred at $(1,0.5,0)$ reciprocal lattice point (RLP) when some atomic SRO is also present.

\section{Sample characteristics and experimental details}

The investigated sample of the $\mathrm{Mn}_{0.4} \mathrm{Cu}_{0.6}$ alloy is a single-crystal grown in the Bardin Central Research Institute for the Iron and Steel Industry, Moscow, Russia. It was grown about 10 years ago and then it was kept at room temperature. The sample is the cube measuring $1 \times 1.2 \times 1.2 \mathrm{~cm}^{3}$ and its mosaic spread is $\eta \approx 40^{\prime}-50^{\prime}$. The crystal structure is fcc with the lattice constant $a=3.767 \AA$ at room temperature. For the alloy of the same Mn content the temperature of the spin-glass freezing is $T_{\mathrm{F}}=156 \mathrm{~K}$ for the quenched powder sample and $T_{\mathrm{F}}=161 \mathrm{~K}$ for the aged powder sample [17]. We assume that freezing temperature of our sample is contained in this temperature range.

The measurements were performed by the 3-axis neutron spectrometers at the MARIA reactor, Świerk. The pyrolytic graphite (PYG) was used as the 
monochromator and analyser. The neutron wavelength was $2.35 \AA$; and $3 \mathrm{~cm}$ PYG filter before the analyser was used to eliminate higher order contamination. Additional measurements were performed using neutrons of the wavelength $1.7 \AA$ in the surrounding of the $(0.5,1,0),(1.5,1,0),(2.5,1,0)$ and $(0.5,3,0)$ RLPs. The measurements for $390 \mathrm{~K}$ were performed with different collimations together with some room-temperature measurements used for the intensity calibration.

\section{Results}

In our $\mathrm{Mn}_{0.4} \mathrm{Cu}_{0.6}$ alloy the broad maxima of the diffuse neutron scattering (DNS) intensity were observed at the $(0.5,1,0) \mathrm{RLP}$ and other points connected to it by symmetry relations. They are similar to those observed at room temperature in $\mathrm{Mn}-\mathrm{Cu}$ alloys with lower $\mathrm{Mn}$ content. The intensity distribution measured at $15 \mathrm{~K}$ in the $(001)$ plane in the vicinity of the $(0.5,1,0)$ is shown in Fig. 1 . The high intensity area is extended in the [100] direction.

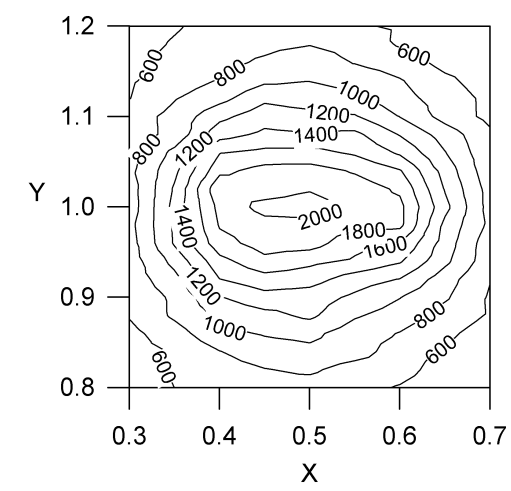

Fig. 1. The scattered neutron intensity distribution $I(Q)$ in the surrounding of the $(0.5,1,0)$ RLP for $15 \mathrm{~K}$.

The observed neutron scattering is caused by both magnetic and atomic contributions. Without polarised neutrons the contributions can be separated due to strong dependence of the magnetic scattering intensity on the scattering-vector length. The magnetic form factor squared at the $(1,2.5,0)$ RLP is lower than that at the $(1,0.5,0)$ RLP by factor lower than 0.08 according to [21].

The measurements of DNS in the surrounding of the $(-2.5,1,0)$ RLP were performed to estimate the atomic SRO. The distributions measured at room temperature for the scans in [100] and [010] directions through the $(2.5,1,0)$ RLP are shown in Fig. 2a and those through the $(-0.5,1,0)$ RLP in Fig. $2 \mathrm{~b}$.

The distribution in the surrounding of the $(1,2.5,0)$ RLP is nearly isotropic in the (001) plane. The shape is Lorentzian with the same full width at half maximum FWHM $=0.33 \pm 0.01 \mathrm{RLU}$ (reciprocal lattice units) in both directions. The resulting atomic correlation range is $\approx 6 \pm 0.2$ lattice constants. 

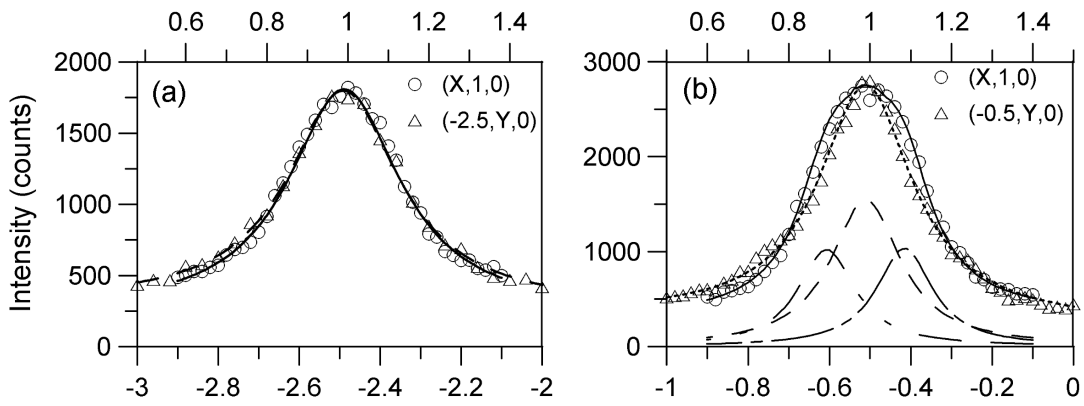

Fig. 2. The intensity distributions measured at room temperature along the $[010]$ and [100] lines through the $(1,2.5,0)$ RLP (a) and those through the $(0.5,1,0)$ RLP (b). The solid and dashed lines in (a) are the fits to the data for the [100] and [010] scans, respectively. The dotted line in (b) is the 1-Lorentzian fit to the data of [010] scan, the solid line is the 3-Lorentzian fit to the data of the [100] scan and the dashed lines are its components.

The shape of the distribution in the surrounding of the $(0.5,1,0)$ RLP in the [010] direction is Lorentzian but its upper part is widened in the [100] direction. This distribution can be fitted by a sum of the 3 Lorentzians with maxima placed along the [100] direction. The significantly higher intensity compared to that at $(2.5,1,0)$ and different shape indicate the significant contribution of the magnetic scattering at $(0.5,1,0)$ at room temperature.

The $I(Q)$ distributions at $(0.5,1,0)$ RLP were measured at 11 temperatures for both the [010] direction (the $(0.5, y, 0)$ scan) and the [100] direction (the $(x, 1,0)$ scan). The temperature dependence of the total integrated intensity of both types of scans is shown in Fig. 3. This total intensity includes the background and the incoherent scattering. The measurements at $390 \mathrm{~K}$ were performed at different conditions than other measurements and the intensity was scaled using the room-temperature data performed for both equipment sets.

Within the temperature range from $15 \mathrm{~K}$ up to $388 \mathrm{~K}$ the total intensity (including background) for $(x, 1,0)$ distributions decreases by a factor 2 and for the $(0.5, y, 0)$ distributions by a factor 1.8 . This decrease is the evidence of the magnetic character of the significant part of the observed intensity. The intensity decrease is observed far above the freezing temperature, since within the spectrometer energy resolution the "inelastic" scattering intensity contains some quasi-elastic contribution due to fluctuations, as expected for a spin glass. A slight temperature dependence of the incoherent scattering is probably caused by disordered freezing of the magnetic moments which do not form any short-range order.

The total intensity of the $(x, 1,0)$ scans is higher than that for the $(0.5, y, 0)$ scans especially at low temperature, which is caused by the anisotropy of the intensity distribution around $(0.5,1,0)$ RLP. The total intensity of the $(x, 1,0)$ 


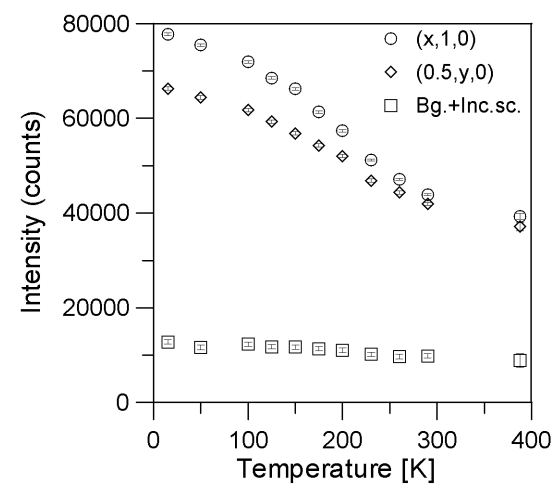

Fig. 3. The temperature dependence of the integrated intensity of the $I(Q)$ scans through $(0.5,1,0)$ RLP along the [100] and [010] directions and the background plus incoherent scattering.

scans contains higher magnetic contribution than that of the $(0.5, y, 0)$ scans. The comparison of the distributions measured at $15 \mathrm{~K}$ and $290 \mathrm{~K}$ for both [010] and [100] directions is presented in Fig. 4.
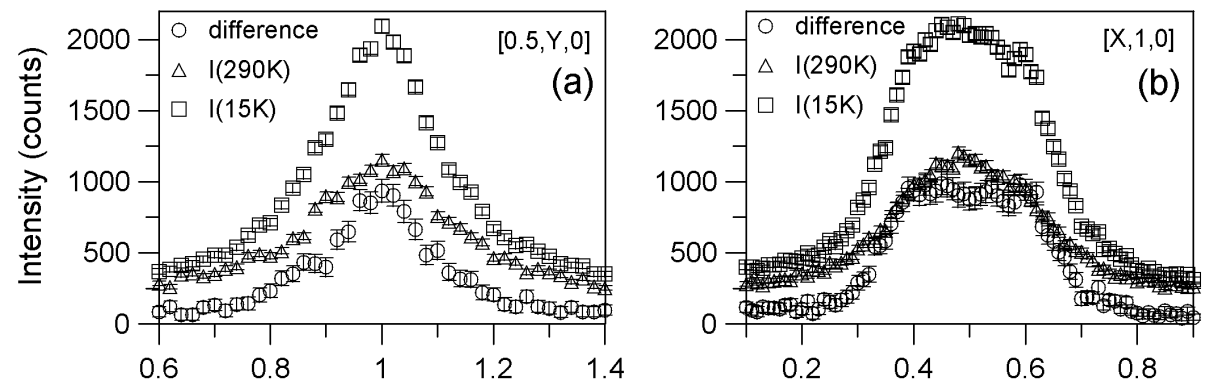

Fig. 4. The intensity distributions $I(Q)$ through the $(-0.5,1,0)$ RLP for $15 \mathrm{~K}$ and $290 \mathrm{~K}$ and their difference measured along the [010] (a) and [100] (b) directions.

The shape of the intensity distribution along the [010] is nearly independent of temperature. It is Lorentzian and its width increases weakly with temperature. The shape of the distribution along the [100] clearly depends on temperature. When the temperature decreases, the upper part of the distribution becomes wider.

The distribution of the difference of the intensities measured along the [100] direction at $15 \mathrm{~K}$ and $290 \mathrm{~K}$ has a wide and a very flat maximum between $(0.4,1,0)$ and $(0.6,1,0)$ RLP. In the shape change of the distribution $(x, 1,0)$ the points $(0.4,1,0)$ and $(0.6,1,0)$ play clearly a special role. At these points the contribution of the magnetic scattering is highest.

The magnetic component of the DNS is separated better by taking the difference between the intensities in the surrounding of the $(1,0.5,0)$ RLP and of the $(1,2.5,0)$ RLP. This is shown in Fig. 5 . 


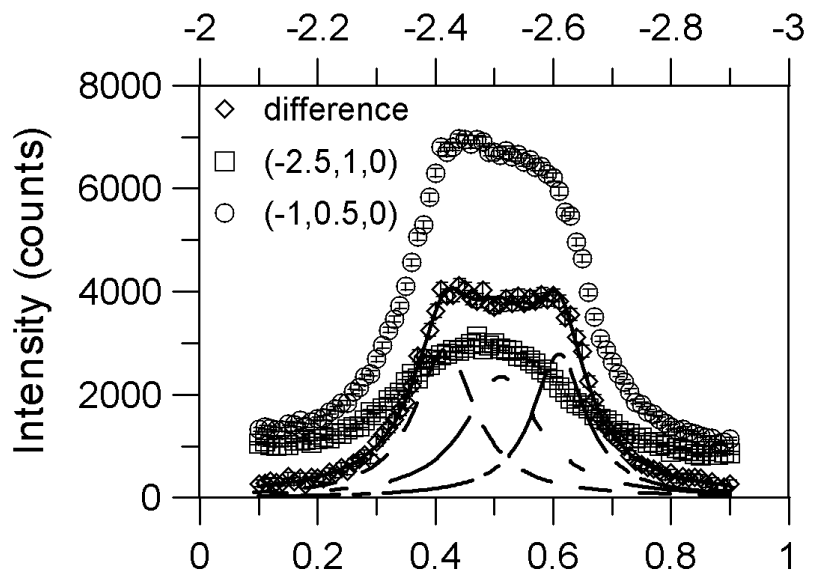

Fig. 5. The intensity distributions $I(Q)$ through the $(-1,0.5,0)$ RLP along the [010] direction and through the $(-2.5,1,0)$ RLP along the [100] for $15 \mathrm{~K}$ and their difference. The 3-Lorentzian fit to the difference data and its components are shown by the solid and dashed lines, respectively.

The shape of the magnetic contribution can be fitted by 3 Lorentzians centred at $(1,0.4,0),(1,0.5,0)$ and $(1,0.6,0)$. Thus we have the magnetic SRO of the incommensurate antiferromagnetic character connected with the $(1,0.4,0)$ and $(1,0.6,0)$ maxima and commensurate magnetic SRO connected with the $(1,0.5,0)$ maximum which could be ferro- or antiferromagnetic. Small-angle neutron scattering was measured in order to answer this question. No significant intensity above the background was observed. Thus there are no magnetic clusters with significant ferromagnetic order. The maximum at $(1,0.5,0)$ RLP is connected to the commensurate antiferromagnetic SRO.

\section{Summary and conclusions}

In our $\mathrm{Mn}_{0.4} \mathrm{Cu}_{0.6}$ sample wide regions of clearly enhanced intensity of the scattered neutrons were observed around the $(0.5,1,0)$ RLP and other points connected to it by symmetry relations of the reciprocal lattice.

The diffuse neutron scattering in the vicinity of $(2.5,1,0)$ and $(0.5,3,0)$ RLP comes mainly from the atomic SRO. The shape of the intensity distributions is very close to the Lorentzian both for the [100] and [010] directions with the same half-widths.

The DNS observed at room temperature in the vicinity of the $(0.5,1,0)$ point is anisotropic and its intensity is by $75 \%$ higher than that for the $(0.5,3,0)$ point. This increase comes from the magnetic scattering which has probably the quasi-elastic paramagnetic character since the room temperature is higher than the assumed $T_{\mathrm{F}}$ by nearly a factor of 2 . The intensity distribution is Lorentzian for the [010] direction while for the [100] direction its peak is wider. It reminds the 
shape of the calculated paramagnetic susceptibility for the disordered $\mathrm{Mn}_{0.85} \mathrm{Cu}_{0.15}$ alloy [22].

The intensity of the scattering measured at the $(0.5,1,0)$ RLP increases nearly by a factor of 2 when the temperature decreases from $388 \mathrm{~K}$ to $15 \mathrm{~K}$. The intensity distributions in the [100] direction have the flattened peaks and they change strongly with temperature. These shapes can be fitted by 3 Lorentzians with the maxima placed along the [100] direction.

The distribution of the difference of the intensities measured along the [100] direction at $15 \mathrm{~K}$ and $290 \mathrm{~K}$ (see Fig. 4) and the distribution of the difference between the intensities measured in the surrounding of the $(1,0.5,0)$ RLP and of the $(2.5,1,0)$ RLP at $15 \mathrm{~K}$ (see Fig. 5) reveal special features at the $(0.4,1,0)$ and $(0.6,1,0)$ RLPs. The intensity maxima at these points are connected with the SRO incommensurate SDW.

In the earlier studies of $\mathrm{Mn}-\mathrm{Cu}$ alloys with a lower $\mathrm{Mn}$ concentration, the diffuse neutron scattering originating from the short range ISDW was observed at points which can be described as $(0.5 \pm \delta, 1,0)$ and a linear dependence of $\delta$ on $x$ was established [6]. This fact was thought to be connected with the increase in the radius of the Fermi sphere for the $4 s$ electrons with the increase in Mn concentration $[6,11]$. The theoretical considerations [22] indicate that in $\mathrm{Mn}_{0.15} \mathrm{Cu}_{0.85}$ alloy, the damped interaction of the Ruderman-Kittel-Kasuya-Yosida (RKKY)-type by the $4 s$ electrons of $\mathrm{Cu}$ is more important than the direct exchange interaction among $3 d$ electrons of $\mathrm{Mn}$. The linear dependence of $\delta$ on Mn concentration confirms these conclusions.

The value of $\delta \cong 0.1$ resulting from our measurements fits well the extrapolation of the linear dependence of $\delta(x)$. The exchange interaction of the damped RKKY-type is still important for the magnetism of the $\mathrm{Mn}_{x} \mathrm{Cu}_{1-x}$ alloys at least up to $x=0.4$.

The "commensurate" magnetic contribution to the neutron scattering (at the $(0.5,1,0)$ RLP) is also present (see Fig. $4 \mathrm{~b}$ and Fig. 5). Since there is no observable intensity of the small-angle neutron scattering, there is no significant amount of ferromagnetic clusters and the above mentioned magnetic contribution has commensurate antiferromagnetic character. This conclusion is consistent with the earlier results for lower $\mathrm{Mn}$ concentration that the correlations of the first Mn neighbours are antiferromagnetic and that of the second ones are ferromagnetic $[1,12-15]$.

The temperature dependence of the neutron-scattering intensity observed far from the maxima indicates the disordered freezing of some local moments not connected to the short-range order.

\section{References}

[1] P. Wells, J.H. Smith, J. Phys. F, Met. Phys. 1, 763 (1971).

[2] S.A. Werner, J.W. Cable, J. Appl. Phys. 3, 1757 (1981). 
[3] J.W. Cable, S.A. Werner, G.P. Felcher, N. Wakabayashi, Phys. Rev. Lett. 49, 829 (1982).

[4] J.W. Cable, S.A. Werner, G.P. Felcher, N. Wakabayashi, Phys. Rev. B 29, 1268 (1984).

[5] T.M. Harders, P. Wells, J. Phys. F, Met. Phys. 13, 1017 (1983).

[5] S.A. Werner, J.J. Rhyne, J.A. Gotaas, Solid State Commun. 56, 457 (1985).

[7] Y. Tsunoda, J.W. Cable, Phys. Rev. B 46, 930 (1992).

[8] J.W. Cable, Y. Tsunoda, J. Appl. Phys. 73, 5454 (1993).

[9] J.A. Mydosh, J. Appl. Phys. 63, 5415 (1988).

[10] Y. Tsunoda, J.W. Cable, Phys. Rev. B 46, 930 (1992).

[11] F.J. Lamelas, S.A. Werner, S.M. Shapiro, J.A. Mydosh, Phys. Rev. B 51, 621 (1995).

[12] T.J. Hicks, J.W. Cable, Phys. Rev. B 58, 5177 (1998).

[13] A.P. Murani, O. Schärpf, K.H. Andersen, D. Richard, R. Raphel, Physica B 267268, 131 (1999).

[14] H. Roelofs, B. Schönfeld, G. Kostorz, W. Bührer, J.L. Robertson, P. Zschack, G.E. Ice, Scr. Materialia 34, 1393 (1996).

[15] B. Schönfeld, R. Bucher, G. Kostorz, M. Zolliker, Phys. Rev. B 69, 224205 (2004).

[16] R.W. Tustison, P.A. Beck, Solid State Commun. 20, 841 (1976).

[17] P. Gibbs, T.M. Harders, J.H. Smith, J. Phys. F, Met. Phys. 15, 213 (1985).

[18] G.E. Bacon, I.W. Dunmur, J.H. Smith, R. Street, Proc. R. Soc. A 241, 223 (1957).

[19] A.I. Bichinashvili, E.Z. Vintaikin, D.F. Litvin, V.A. Udovenko, Fiz. Met. Metallov. 41, 130 (1976).

[20] E.Z. Vintaikin, V.B. Dmitriev, V.A. Udovenko, Fiz. Met. Metallov. 44, 1023 (1977).

[21] C.G. Shull, W.A. Strauser, E.A. Wollan, Phys. Rev. 83, 333 (1951).

[22] M.F. Ling, J.B. Staunton, D.D. Johnson, J. Phys., Condens. Matter 6, 6001 (1994). 